\title{
Papillary Renal Carcinoma Presenting as a Cancer of Unknown Primary (CUP) and Diagnosed through Gene Expression Profiling
}

\author{
Steven M. Sorscher ${ }^{a}$ Frank Anthony Greco ${ }^{b}$ \\ ${ }^{a}$ Oncology Section, Department of Medicine, Washington University, \\ St. Louis, Mo., and ${ }^{\mathrm{b}}$ Tennessee Oncology, PLLC, Nashville, Tenn., USA
}

\section{Key Words}

Occult primary · Gene expression profiling · Cancer of unknown primary · Papillary renal carcinoma

\begin{abstract}
Cancer of unknown primary (CUP) is a clinical syndrome representing many types of cancers and diagnoses are typically made after review of clinical presentation, pathology (including immunohistochemical staining) and imaging studies. Treatment with systemic chemotherapy has been shown to result in fairly reproducible objective response rates. Herein, a case of a patient who was initially diagnosed with a poorly differentiated adenocarcinoma of unknown origin is reported. After mRNA gene expression profiling (commercially available CancerTYPE ID), a specific diagnosis of papillary renal cell carcinoma (RCC) was made and then confirmed with additional immunohistochemical staining. The patient was treated with targeted therapy and an objective radiographic response was seen. A literature review suggests this to be the first patient with papillary RCC, identified by molecular profiling, and benefitting from a targeted agent that otherwise would not have been considered in the setting of CUP. This case underscores the importance of considering the use of newer testing technologies in the interest of offering patients more specific, targeted therapy in order to improve efficacy and spare patients toxicities of less specific, empiric chemotherapeutic regimens.
\end{abstract}




\section{Introduction}

Various guidelines have been suggested for appropriate evaluation of metastatic cancers when the primary tumor site is not readily identifiable based on the clinical presentation, tissue histology and immunohistochemical staining. After such evaluation, cancer of unknown primary (CUP) is the diagnosis made in about $4-5 \%$ of invasive cancers [1]. Series of patients with this diagnosis have been studied and treated with empiric combination chemotherapy regimens. Both the specific recommended regimens and the efficacy of these therapies vary by the histologic subtype and immunohistochemical and clinical presentation [2]. Objective response rates in the range of $30-50 \%$ have been described in many published series [3]. For example, in a series of 71 patients treated with paclitaxel, carboplatin and oral etoposide, $46 \%$ of patients had a major objective response with a median survival of 11 months [4].

The National Comprehensive Cancer Network (NCCN) has published guidelines with recommendations concerning evaluation and care for patients with CUP [2]. Molecular profiling is described as an 'emerging diagnostic tool', with the potential of identifying the likely primary based on comparison of expression patterns in the unknown tumor relative to known data banks that include known primaries [5-10]. Confirming the specificity of the testing in a particular patient remains challenging, if not impossible, short of autopsy series; however, with improved chemotherapy and targeted therapies now available for several solid tumors, a more site-specific therapy is likely to be superior for CUP patients diagnosed with a specific tissue of origin compared to the administration of an empiric broad-spectrum chemotherapy regimen. Ultimately, the recognition of specific molecular abnormalities (mutations) in tumor cells which can be targeted with specific drugs is likely to be far more clinically relevant than even identifying the primary tissue of origin in terms of choosing therapies. Herein, we describe a patient initially diagnosed with CUP. Expression profiling strongly suggested the tissue of origin to be the kidney and the patient's tumor responded well and durably to a targeted agent which is FDA approved for treating metastatic kidney cancer.

\section{Case Report}

PG is a 53-year-old male who presented in April 2011 with right hip pain. Plain films revealed osteoarthritis and physical examination showed left neck lymphadenopathy. CT, PET/CT and MRI scanning confirmed diffuse lymphadenopathy with the PET scan showing markedly increased FDG uptake involving the left neck and supraclavicular regions (left supraclavicular lymph node measuring $4.1 \times 2.6 \mathrm{~cm}$ ), mediastinal, retroperitoneal, bilateral and common external and internal iliac lymph node chains. The MRI report noted 'extensive retroperitoneal' and 'retrocrural adenopathy' as well as a large necrotic mass apparently 'invading' the right kidney, and an adrenal gland felt also to represent 'metastatic nodes'. Left supraclavicular lymph node biopsy showed a 'poorly differentiated adenocarcinoma'. Immunohistochemical staining was done including negative staining for cytokeratin 20, TTF-1, calretinin, CA19-9, WT-1 and D2-40, and strongly positive staining for cytokeratin 7. The phenotype was felt to be consistent with a pancreatobiliary, lung or upper GI primary site.

The pathologists wrote 'the morphology does not suggest a renal or adrenal primary', a question that had been raised based on the scans above. CEA, HCG and AFP were normal, while CA19-9 was 64.9 units/ml (normal range 0-35) and LDH was 214 IU/l (98-192). 
The tumor was sent for mRNA expression profiling [11] (bioTheranostics, San Diego, Calif., USA) and the report suggested a 95\% 'probability' of this cancer representing kidney cancer with a 94\% 'probability' of the tumor being a papillary renal cell carcinoma (RCC). Because of the bioTheranostics expression profiling result, further immunostaining was done. The tumor stained strongly positive for racemace (AMACR), RCC antigen and CD10. The pathologist concluded that the immunohistochemical profile strongly supports the tumor to be renal in origin and specifically papillary renal carcinoma'.

The patient was treated with everolimus (10 mg po qd) beginning on May 10, 2011, and, 2 weeks later, reported a diminution in the supraclavicular fullness. On June 23, 2011, a pelvic MRI was repeated and the report noted a mild decrease in the size of the retroperitoneal adenopathy. On July 5 , 2011, a PET/CT was interpreted as showing relatively decreased FDG uptake in multiple lymph node regions 'suggestive of some interval treatment response' since the prior PET/CT. In March 2012, scans continued to show no evidence of progression of his malignancy.

\section{Discussion}

With improved chemotherapy and targeted therapies now available for several solid tumors, a more site-specific therapy is likely to be superior for CUP patients diagnosed with a specific tissue of origin compared to the administration of an empiric broadspectrum chemotherapy regimen. In the case described above, kidney cancer was identified as the likely cancer type through expression profiling, and the patient was successfully treated with a targeted agent indicated for treatment of that cancer type.

Treating some CUP patients with systemic therapies described for known counterpart primaries based on gene expression profiling has resulted in more favorable outcomes compared to those outcomes reported for historic controls of CUP patients treated with the less specific regimens, where no expression profiling was completed. For example, Greco et al. [12] recently noted a 64\% RR and a 22-month median survival for 32 CUP patients identified by expression profiling as having probable colorectal primaries (although colonoscopies were negative in 30 of the 32 patients), while historic controls with CUP treated with CUP regimens, rather than colorectal regimens, had a 9-month median survival. Still, no prospective randomized studies have been done and, in spite of small series such as the one above, it may be that while a molecular profile can be consistent with that of a particular primary, the response to available therapies may be different for these tumors, since the biological behavior of CUP may be different from their more readily identifiable counterparts. The NCCN panel concluded 'the panel does not recommend molecular profiling as part of routine evaluation' and suggests no specific circumstances in which such testing should be considered.

\section{Conclusions}

After reviewing the literature, this case appears to represent the first case of a papillary renal carcinoma where expression profiling was used to identify the primary, more specific immunostaining was used to confirm the diagnosis, and the patient was treated with a targeted agent, which resulted in a response. Everolimus is an FDA approved targeted agent for the treatment of advanced renal carcinoma based on a study showing that, compared to placebo, everolimus more than doubled the time without tumor growth or death [13]. Such therapy clearly would not have been a consideration if not for the pursuit of the molecular profiling. Our patient's tumor 
initially responded to everolimus and has shown no progression for over 10 months. While choosing which specific patients will benefit from expression profiling remains problematic, cases such as this highlight the importance of considering such testing in CUP patients.

\section{References}

1 Greco FA, Hainsworth JD: Introduction: unknown primary cancer. Semin Oncol 2009;36:6-7.

2 National Comprehensive Cancer Network: Clinical Practice Guidelines in Oncology. Occult Primary (Cancer of Unknown Primary [CUP]) 2011.

3 Greco FA, Hainsworth JD: Cancer of unknown primary; in DeVita VT Jr, Lawrence TS, Rosenberg SA (eds): Cancer Principles and Practice of Oncology, ed 9. Philadelphia, Lipincott Williams and Wilkens, 2011 pp 2033-2051.

4 Greco FA, Burris HA 3rd, Erland JB, et al: Carcinoma of unknown primary site. Cancer 2000;89:26552660.

5 Bender RA, Erlander MG: Molecular classification of unknown primary cancer. Semin Oncol 2009;36:3843.

6 Monzon FA, Koen TJ: Diagnosis of metastatic neoplasms: molecular approaches for identification of tissue of origin. Arch Pathol Lab Med 2010;134:216-224.

7 Talantov D, Baden J, Jatkoe T, et al: A quantitative reverse transcriptase-polymerase chain reaction assay to identify metastatic carcinoma tissue of origin. J Mol Diagn 2006;8:320-329.

8 Varadhachary GR, Talantov D, Raber MN, et al: Molecular profiling of carcinoma of unknown primary and correlation with clinical evaluation. J Clin Oncol 2008;26:4442-4448.

9 Ma XJ, Patel R, Wang X, et al: Molecular classification of human cancers using a 92 -gene real-time quantitative polymerase chain reaction assay. Arch Pathol Lab Med 2006;130:465-473.

10 Greco FA, Spigel DR, Yardley DA, et al: Molecular profiling in unknown primary cancer: accuracy of tissue of origin prediction. Oncologist 2010;15:500-506.

11 Erlander MG, Ma XJ, Kesty NC, et al: Performance and clinical evaluation of the 92-gene real-time PCR assay for tumor classification. J Mol Diagn 2011;13:493-503.

12 Greco FA, Lennington WJ, Spigel DR, et al: Carcinoma of unknown primary site (CUP): outcomes in patients with a colorectal molecular profile treated with site-specific chemotherapy. ASCO Meeting Abstracts 2011;29:3563.

13 Motzer RJ, Escudier B, Oudard S, et al: Efficacy of everolimus in advanced renal cell carcinoma: a doubleblind, randomised, placebo-controlled phase III trial. Lancet 2008;372:449-456. 